\title{
Characterisation of bioactive protein-bound polysaccharides from Amanita ponderosa cultures
}

Salvador, C. ${ }^{1,2,4}$; Martins, M.R. ${ }^{1,3,4}$; Arteiro, J.M. ${ }^{2}$; Caldeira, A.T. ${ }^{1,2,4}$

${ }^{1}$ Departamento de Química, Universidade de Évora, Rua Romão Ramalho 59, 7000 - 671 Évora, PORTUGAL

${ }^{2}$ Centro de Química de Évora (CQE), Universidade de Évora, Rua Romão Ramalho 59, 7000 - 671 Évora, PORTUGAL

${ }^{3}$ Instituto de Ciências Agrárias e Ambientais Mediterrânicas (ICAAM), Universidade de Évora, Núcleo da Mitra Apartado 94, 7002-554 Évora, PORTUGAL

${ }^{4}$ Laboratório HERCULES, Universidade de Évora, Largo Marquês de Marialva 8, $7000-809$ Évora, PORTUGAL

Email: atc@uevora.pt ; Katia_salvador@hotmail.

Different bioactive compounds of edible mushrooms are responsible for their antioxidant, antitumor, antimicrobial, immunomodulatory, antiatherogenic and hypoglycemic reported properties [1, 2]. These properties are mostly due to the ability to synthesize different polysaccharides, namely proteinpolysaccharide (PPS) complexes. The antioxidant capacity of these compounds present great interest in preventing innumerous diseases, including cancer, cardiovascular, auto-immunes diseases and accelerated aging. Amanita ponderosa are wild edible mushroom (Fig $\left.1 \mathrm{a}_{1}, \mathrm{a}_{2}, \mathrm{~b}\right)$, growing in some Mediterranean microclimates, namely in Alentejo region (Southern Portugal) and Andalusia (Southern Spain) [3], and establishes a mycorrhizal symbiosis with holm oaks and cork trees like Quercus ilex and $Q$. suber. There are few studies with respect to this species, however in this work was possible to obtain A. ponderosa pure cultures (Fig 1 c, $d_{1}, d_{2}$ ) from strains collected in different areas of Alentejo.

This study focused on the characterisation of the PPS complexes produced in liquid cultures of A. ponderosa. Batch cultures (Fig 1e $\mathrm{e}_{1}, \mathrm{e}_{2}$ ) were performed during 15 days, and polysaccharides concentrations were determined by the phenol-sulphuric method. A combined FTIR-ATR (Fourier-transform infrared using the attenuated total reflection) and Raman spectroscopy was used for the screening of bioactive PPS compounds present in the culture extracts. After identification of these bioactive compounds, PPS extracts were fractionated by size exclusion chromatography (SEC) using Sephacryl S-300 as stationary phase. Then, the chromatographic fractions and extracts were analysed by SEC, using an HPLC system coupled to UV (280 $\mathrm{nm})$ and RI detectors in order to determine polysaccharide average molecular weights $(\mathrm{Mw})$. The toxicity of the dried mushrooms, cultures and PPS extracts was assessed using Artemia salina test kit (Artoxkit MTM, Microbiotest). Acute toxicity was evaluated in vivo using Swiss mice. Samples of dried fruiting bodies and lyophilized mycelia were orally administered, by means a gastric probe, at a concentration of $5000 \mathrm{mg} \mathrm{kg}^{-1}$ and $\mathrm{LD}_{50}$ was evaluated.

The aim of this study was the purification and characterisation of PPS complexes produced by A. ponderosa cultures using a new microanalytical approach to monitoring the production, in situ. Microanalysis using FTIR-ATR (Fig. 2) and Raman of PPS samples showed spectra compatible with identification of this type of compounds in culture extracts [4]. IR band between $3000-3500 \mathrm{~cm}^{-1}$ arise from $\mathrm{OH}$ stretching vibrations of hydroxyls and water, moderate IR bands between $1600-1400 \mathrm{~cm}^{-1}$ arise from stretching vibrations of carboxylate. Strong IR/Raman bands in the region of $900-1200 \mathrm{~cm}^{-1}$ are characteristic for carbohydrates and can be attributed to coupled $\mathrm{CO}$ and $\mathrm{CC}$ stretching and $\mathrm{COH}$ bending vibrations in polysaccharides. PPS separated by size exclusion chromatography (SEC) showed seven mainly complexes with molecular weights 
ranging between 1.5-20 kDa. Dried fruiting bodies, lyophilized mycelia and PPS extracts did not present toxicity against $A$. salina. Mycelia of cultures and fruiting bodies also did not show toxicological effects in Swiss mice with $\mathrm{LD}_{50}>5000 \mathrm{mg} \mathrm{Kg}^{-1}$.

PPS compounds have displayed important antioxidant properties and further studies are in progress in order to investigate their antitumural activities. The application of microanalytical approach to monitoring the production of PPS compounds can be successful applied in biotechnological processes.

Cátia Salvador acknowledges Fundação para a Ciência e a Tecnologia for financial support (PhD grant, SFRH/BD/61184/2009) through program QREN-POPH-typology 4.1., co-participated by the Social European Fund (FSE) and MCTES National Fund.

[1] Arteiro, J.M. et al., Medicinal Chemistry Research, 2, 937-943, 2012.

[2] Salvador, C. et al., Journal of Agricultural Science and Technology A, 2, 1296-1306, 2012.

[3] Salvador, C. et al., Agroforestry Systems, 87, 295-302, 2013.

[4] Na, Y.S. et al., International Immunopharmacology, 10, 364-370, 2010.

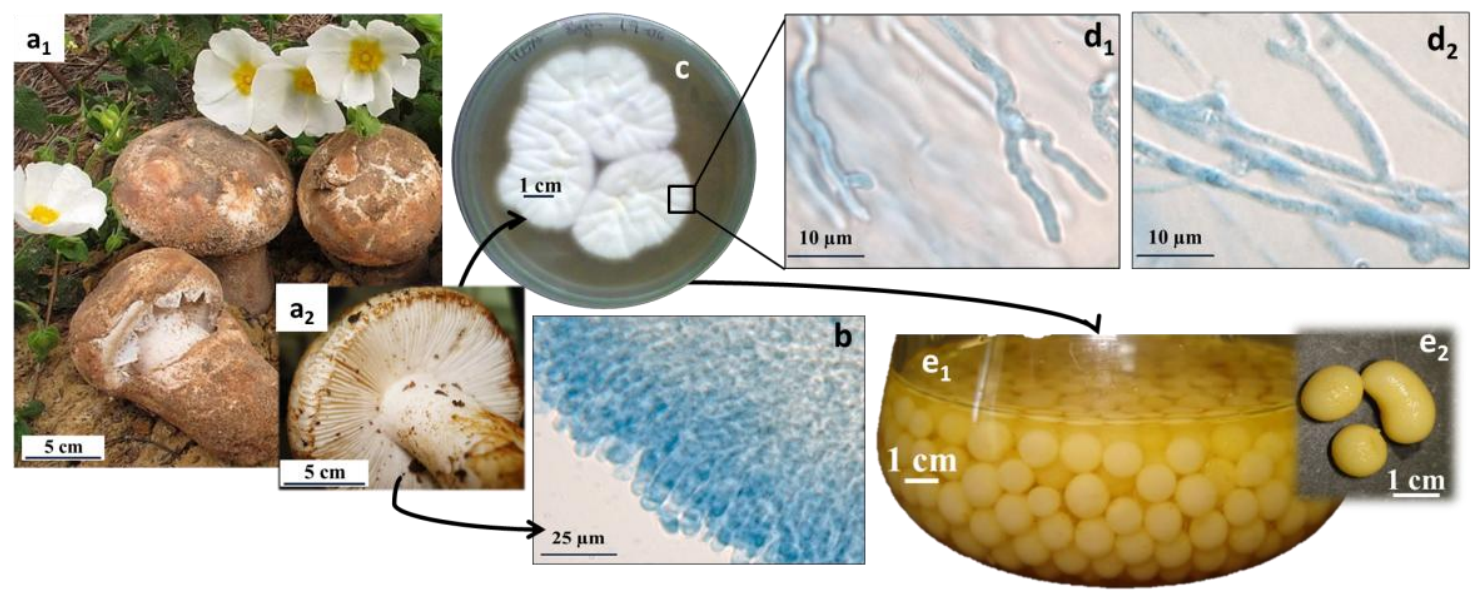

Figure 1: $\mathrm{a}_{1}$ and $\mathrm{a}_{2}$ ) Macroscopic features of wild edible mushrooms $A$. ponderosa, b) micromorphological features of $A$. ponderosa hymenium with basidia, c) the mycelium of $A$. ponderosa pure cultures in solid medium, $\mathrm{d}_{1}$ and $\mathrm{d}_{2}$ ) micromorphological features of mycelium with observation of septate hyphae forming a "fork-like" characteristic structures in the end regions and clamp connections, $\mathrm{e}_{1}$ and $\mathrm{e}_{2}$ ) liquid cultures of $A$. ponderosa mycelium.

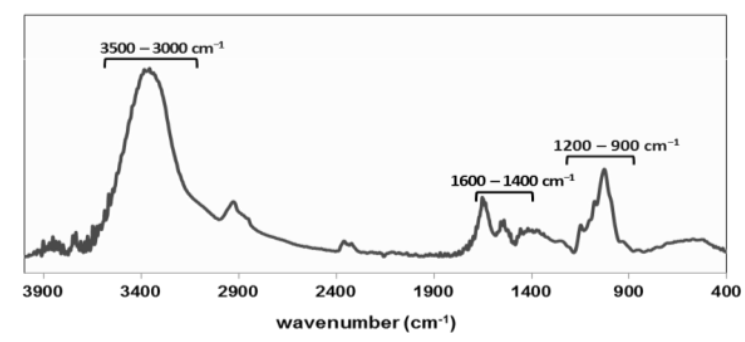

Figure 2: FITR-ATR spectrum of protein-polysaccharide complexes obtained from cultures. 